\title{
Understanding tight junction clinical physiology at the molecular level
}

\author{
Bruce R. Stevenson
}

Department of Cell Biology, University of Alberta, Edmonton, Alberta T6G 2H7, Canada

Address correspondence to: Bruce R. Stevenson, Department of Cell Biology, Edmonton, Alberta T6G 2H7, Canada. Phone: (780) 492-1841; Fax: (780) 492-0450; E-mail: Bruce.Stevenson@ualberta.ca.

A century of investigation has led to a reasonable understanding of the role the tight junction (zonula occludens) plays in epithelial physiology $(1,2)$. This intercellular junction forms a regulated, semipermeable barrier in the spaces between epithelial and endothelial cells (the paracellular space). It also acts as a fence, delimiting the compositionally distinct apical and basolateral plasma membrane domains characteristic of these cell types. Both the barrier and fence functions, in conjunction with transcellular vectorial transport processes, permit the establishment of unique environments in opposing compartments separated by epithelial cell sheets. Interaction and exchange between these environments is critical to normal organ biology. Moreover, integrity of compartmental boundaries is key to protecting an organism from noxious agents present in the outside world.

Our knowledge of the molecules that make up the tight junction is less developed, dating back slightly over a decade. While we are aware of 9 peripheral and 3 integral membrane proteins (Table 1), we have only just started to obtain isolated and limited information on the possible functions of individual molecules $(3,4)$. However, in this issue of the JCI, Wan et al. (5) establish the first direct link between a clinically relevant issue the access of asthma-inducing dust mite allergen to the immune system and a specific molecular event at the tight junction. This paper is a landmark in the molecular understanding of the tight junction and the clinical physiology of epithelia.

Tight junction molecules and regulation of paracellular permeability. The proteins peripherally associated with the tight junction membrane include $\mathrm{ZO}-1, \mathrm{ZO}-2$, and ZO-3, members of a larger protein family known to be involved in the organization of supramolecular complexes at specific areas of the cell surface (3). These proteins are capable of interacting directly with the cytoplasmic domain of occludin, a 4-passage transmembrane protein present at the tight junction (6). Most recently, a family of transmembrane elements, the claudins, have been identified (7). Members of the claudin family, now totaling 16 individual proteins, are present in varying amounts in different tissues, suggesting that expression of various claudins relates to the distinct paracellular permeability properties observed in different epithelia or endothelia (4).

Although we know that permeability through the tight junction is regulated in a physiologically relevant manner (2), the molecular mechanisms underlying this regulation are unresolved. It is widely assumed that actin filaments found at the tight junction participate in junctional regulation (2), and actin may be linked to occludin through the $\mathrm{ZO}$ proteins $(8-10)$. While there is evidence that occludin acts in the regulation of the junctional barrier (11), it is likely that formation and regulation of the paracellular barrier and intramembrane fence require claudins and occludin acting in concert. It is also clear that the tight junction can be regulated from within the cell (2), as well as by externally applied factors both artificial (e.g., synthetic peptides; ref. 12) and natural (e.g., cholera toxin; ref. 13). However, prior to the results presented here, there have been no data directly linking the effects of a natural external agent and a tight junction molecule.

Dust mite allergen and the tight junction: Where are we? Where are we going? Wan et al. (5) demonstrate that treatment of epithelial cell lines with either dust mite fecal pellets or Der $p$ 1 , a protease purified from these pellets, reversibly disrupts tight junctions. Moreover, this disruption leads to increased paracellular movement of the Der $\mathrm{p} 1$ allergen across the epithelial boundary. Such transepithelial transit in a respiratory system in vivo would increase exposure of the host immune system to this allergen, potentially explaining allergic sensitization and subsequent asthma development. Most importantly, the authors provide evidence indicating that Der p 1 acts to increase paracellular permeability by direct proteolytic cleavage of occludin and possibly claudin. This proteolysis is assumed to then cause the breakdown of the tight junction protein complex.

This information raises questions and avenues for further investigations of both the dust mite allergen system and the molecular mechanisms operating at tight junctions. The data indicate that both occludin and claudin are proteolyzed by Der $\mathrm{p} 1$, although it will be important to demonstrate that claudin is acted on directly. Must both proteins be cleaved to open the junction, or is degradation of one or the other sufficient? How does Der p 1 gain access to the extracellular portion of either protein, given the close proximity of cell-cell interactions presumed at junctional contact sites? And does Der $\mathrm{p} 1$ act on JAM, a third transmembrane protein found at the tight junction (14)? Since JAM participates in

\begin{tabular}{lc} 
Table 1 & \\
Tight junction proteins & \\
${ } }$ & Integral \\
ZO-1 & occludin \\
cingulin & JAM \\
ZO-2 & claudins \\
7H6 & \\
Rab3B & \\
symplekin & \\
AF-6 & \\
ZO-3 & \\
ASIP & \\
\hline
\end{tabular}


monocyte transmigration across endothelial monolayers, the results provided (5) suggest a mechanism by which cells migrating across endothelia might utilize cell surface proteases to "chew" their way through tight junctions. How does cleavage of a transmembrane element signal the dissolution of the cytoplasmic complex of junctional proteins? Again, are both occludin and claudins involved in such signaling? Although some information is available on the cytoplasmic binding partners of occludin (3), no information on molecular interactions involving the claudins is currently available. Finally, the results of Wan et al. (5) engender the exciting possibility that tight junction permeability can be modulated for clinical means. Could inhibition of Der $\mathrm{p} 1$ proteolysis in the respiratory system be used to decrease allergic sensitization? Alternatively, could controlled proteolysis or agents targeted to the extracellular segments of occludin, claudins, or JAM be effective in reversibly opening tight junctions at sites where increased drug delivery would be advantageous, such as the intestine or blood-brain barrier?

Like all good science, the results presented by Wan et al. (5) generate as many questions as they answer. The fun lies in the ongoing pursuit of these questions.

1. Balda, M.S., and Matter, K. 1998. Tight junctions. J. Cell Sci. 111:541-547.

2. Madara, J.L. 1998. Regulation of the movement of solutes across tight junctions. Annu. Rev. Physiol. 60:143-159.

3. Stevenson, B.R., and Keon, B.H. 1998. The tight junction: morphology to molecules. Annu. Rev. Cell Dev. Biol. 14:89-109.

4. Goodenough, D.A. 1999. Plugging the leaks. Proc. Natl. Acad. Sci. USA. 96:319-321.

5. Wan, H., et al. 1999. Der p 1 facilitates transepithelial allergen delivery by disruption of tight junctions. J. Clin. Invest. 104:123-133.

6. Furuse, M., et al. 1993. Occludin: a novel integral membrane protein localizing at tight junctions. J. Cell Biol. 123:1777-1788.
7. Morita, K., Furuse, M., Fujimoto, K., and Tsukita, S. 1999. Claudin multigene family encoding four-transmembrane domain protein components of tight junction strands. Proc. Natl. Acad. Sci. USA. 96:511-516.

8. Itoh, M., Nagafuchi, A., Moroi, S., and Tsukita, S. 1997. Involvement of ZO-1 in cadherin-based cell adhesion through its direct binding to a catenin and actin filaments. J. Cell Biol. 138:181-192.

9. Itoh, M., Morita, K., and Tsukita, S. 1999. Characterization of ZO-2 as a MAGUK family member associated with tight as well as adherens junctions with a binding affinity to occludin and alpha catenin. J. Biol. Chem. 274:5981-5986.

10. Wittchen, E.S., and Stevenson, B.R. 1998. Novel binding interactions among tight junction proteins. Mol. Biol. Cell. 9:82a. (Abstr.)

11. Matter, K., and Balda, M.S. 1999. Occludin and the function of tight junctions. Int. Rev. Cytol. 186:117-146.

12. Wong, V., and Gumbiner, B.M. 1997. A synthetic peptide corresponding to the extracellular domain of occludin perturbs the tight junction permeability barrier. J. Cell Biol. 136:399-409.

13. Fasano, A., et al. 1991. Vibrio cholerae produces a second enterotoxin, which affects intestinal tight junctions. Proc. Natl. Acad. Sci. USA. 88:5242-5246.

14. Martin-Padura, I., et al. 1998. Junctional adhesion molecule, a novel member of the immunoglobulin superfamily that distributes at intercellular junctions and modulates monocyte transmigration. J. Cell Biol. 142:117-127. 